\title{
Embedded discontinuity finite element modeling of fluid flow in fractured porous media
}

\author{
Craig D.Foster • Talisa Mohammad \\ Nejad
}

Received: date / Accepted: date

\begin{abstract}
In many geomaterials, particularly rocks and clays, permeability is greatly enhanced by the presence of fractures. Fracture sets create an overall permeability that is anisotropic, enhanced in the directions of the fractures. In modeling the fractures via a finite element method, for example, meshing around these fractures can become quite difficult and result in computationally intensive systems. In this article, we develop a relatively simple method for including the fractures within the elements.

Flow through the bulk medium is assumed to be governed by Darcy's law, and the flow on the fracture by a generalization of the law. This model is embedded in a finite element framework, with the fractures passing through the elements. In this formulation, elements with fractures are given an enhanced permeability in the direction of the fractures. With these enhancements, the material essentially becomes anisotropically more permeable in the direction of fracture sets.
\end{abstract}

Keywords Porous flow · Darcy's Law · Fractured flow · Finite elements

\section{Introduction}

There are many instances of fluid flowing through porous solid media. Groundwater flow, oil and natural gas flow in underground reservoirs, geological fault systems, permeation of concrete, and fluids flowing in biological tissues are examples. In all but the last example, the permeability of the system is often greatly affected by localized structures such as fractures, shear bands, dilation bands, and compaction bands. While opening fractures and dilation bands

C. Foster and T. Mohammad Nejad

University of Illinois at Chicago, Chicago, IL, USA

Tel.: +1-312-9968086

Fax: +1-312-9962426

E-mail: fosterc@uic.edu 
tend to increase fluid flow along the band (16), compaction bands inhibit flow across the band (15). Some systems, like faults, exhibit more complex behavior, with bands of high permeability outside the fault core due to fracture, and very low permeability across to cataclasis of the particles in the band $(1 ; 14)$. The result is both increased permeability in the direction of the fault and impaired permeability across it. In this article we will focus on permeability enhancement in the direction of flow. Modeling the inhibition across the localized regions is saved for another article.

A number of models have been introduced for fluid flow in porous media. At the continuum scale, Darcy's Law is one of the simplest and most widely used. It is an effective model for a wide variety of soils and other materials. In highly permeable materials, where flow may become nonlinear or even turbulent, it may not be accurate. Darcy-Stokes (e.g. (9), among others) or other models may be appropriate. Similarly, modification have been made for clays and other low permeability materials, particularly where the chemical interaction between clay and water molecules becomes a factor. In the paper we will assume Darcy's Law is valid. For complex geometries, a number of numerical techniques can solve the resulting equations in standard ways. The finite element method is the approach we employ in the paper.

There are at least two approaches modeling the effect of localized regions on permeability. If there are dense fractures in the medium, modeling each one may prove to be impractical, and the effect could be incorporated into anisotropy of the permeability matrix. Double-porosity models, proposed by Barneblatt (2), which can differentiate between fluid in more permeable fracture sets and less permeable pores, capture fluid flow better than standard single-porosity models in these situations $(4 ; 19)$. In this article, we are interested in the case of modeling a few, large fractures or other localized zones explicitly. In a finite element context, these fractures may either be meshed explicitly with the bulk material around them, or an intraelement method may be used. While the first approach is certainly viable, there is extra difficulty in meshing around the fractures. The approach is taken in discrete fracture network (DFN) models, for example (17). Some of these models, e.g (11), neglect bulk permeability, which is appropriate in some applications. While there is significant research in this area, the process is computationally intensive.

Intraelement methods initially were developed for problems of localization in mechanics. These approaches include enhanced strain methods, e.g. $(7 ; 8 ; 12)$, extended finite elements $(3)$, and several other formulations. They have been used to model propagating fractures, shear bands, and other localized structures. It is worth noting, too, that some of these methods have been extended to the case of poromechanics $((5 ; 13)$ and references therein), incorporating the effects of fluid flow coupled with solid mechanics.

The focus of this article, however, is to develop an approach just for the evaluation of flow through porous media. As in the methods above, we will assume that the thickness of the fractures is small in comparison to the overall scale of interest. Hence, it will be acceptable to treat the localized region as a line element in two dimensions or a surface element in three. We will develop 
enhancements to the permeability matrix due to the presence of fractures, and then implement those enhancements into a finite element framework. This article builds on the formulation presented in (6).

The remainder of the paper is organized as follows: Section 2 will develop the continuum equations for flow with enhanced permeability in localized regions. Section 3 will discuss how those are implemented into the numerical equations. Section 4 will show some example problems, and finally conclusions will be presented in Section 5 .

\section{Governing equations for porous flow in localized media}

We consider a saturated porous medium with fluid flowing through it. The bulk permeability follows Darcy's Law, which we will write as $\boldsymbol{v}_{b u l k}=-\boldsymbol{k}_{b u l k} \boldsymbol{\nabla}$, where $\boldsymbol{v}_{b u l k}$ is the superficial velocity, $\boldsymbol{k}_{\text {bulk }}$ the permeability in the bulk material, and $h$ the total head. As is standard in this formulation, we assume that the flow velocity is small and ignore the velocity head.

Next we add a fracture or set of fractures or other localized regions to the medium As mentioned previously, these regions have an implied width that is much less than the width of the overall structure. Hence, we will model these regions as being one dimension lower than the structure in which they are embedded, and having an implied enhancement to permeability. We will use the term fractures from here on out, with the understanding that this implies to any localized band with enhanced permeability along its direction.

The enhancement to the permeability will also follow a Darcy-like formulation, with

$$
\hat{\boldsymbol{v}}=-\hat{k}(H) \hat{\boldsymbol{i}}
$$

where quantities with the ${ }^{\wedge}$ indicate enhancements of the permeability due to the fractures. Hence, we can write $\hat{\boldsymbol{i}}=(\boldsymbol{\nabla} h \cdot \boldsymbol{l}) \boldsymbol{l}$ where $\boldsymbol{l}$ is the unit vector in the direction of the fracture. Here, $H$ is the thickness of the fracture surface or deformation band. Clearly, the enhancement of the permeability will vary with crack dilation, though we will not investigate the nature of this relationship in this article. The enhancement to the permeability is only active on the fracture, hence the total flow velocity on the fracture can be written

$$
\boldsymbol{v}=\boldsymbol{v}_{b u l k}+\hat{\boldsymbol{v}}=-\boldsymbol{k}_{b u l k} \boldsymbol{\nabla} h-\hat{k}(H) \hat{\boldsymbol{i}} \delta_{S}
$$

where $\delta_{S}$ is a Dirac delta function acting on the surface.

\subsection{Differential Equations}

The governing differential equation, in this form, is not much different than the continuum case. 


$$
\boldsymbol{\nabla} \cdot \boldsymbol{v}+G=0
$$

Where $G$ is a fluid source term, such as a fluid injection site. When we assume the validity of Darcy's Law

$$
\boldsymbol{v}=-\boldsymbol{k} \boldsymbol{\nabla} h
$$

the equations can be solved. The difference lies only in the modifications to the terms $\boldsymbol{k}$.

\section{Finite element implementation}

The differential equation is solved approximately using the finite element method. We choose total head $h$ as a primary variable. Other researchers have, equivalently, tracked pore pressure. To accommodate the enhancements, we add nodes to the element where the fracture surface intersects the element edges. By connecting these nodes, the element becomes divided. While many techniques have been developed to handle this division, the simplest for the case is to treat each subelement separately and perform the integration on each side, as shown in Figure 1. There are a few ways these divisions can be accomplished. For simplicity, we do not add nodes to the band inside the element, allowing a piecewise linear gradient on the fracture surfaces. While not necessary, this approach simplifies the formulation. Since the gradient along the fracture surface will be treated as linear, using high-order interpolation in the bulk elements will not improve the rate of convergence.

Of the possible ways to split the element, we choose to split an initially triangular element. The advantage of starting with triangular elements is that there will always be one side with one of the initial nodes and the other side with two. We choose to treat this second side as a quadrilateral sub-element, as in Figure 1a, though it could also split into two triangular sub-elements (Figure 1b). The resulting shape functions (Figure 2) follow the shape functions for each subelement type. If the corresponding node is not part of both sub-elements, the shape function is zero on the sub-element it does not correspond to. If it is on both sub-elements, it uses the linear shape function for the triangular sub-element and bilinear for the quadratic shape function. Quadrilateral elements can also be divided, but with different results depending how the element is split. If two nodes are on each side of the fracture, as shown in Figure 1c, two quadrilateral subelements may be used. If one corner is cut off, as in Figure 1d, the triangular subelement may be used on the corner side, and the side with three nodes may be divided into three separate triangles. These are not the only options, but the case is more complex than for triangles.

Elements at the ends of the fracture may simply be split into two triangular sub-elements, as shown in Figure 3. These elements do not contain the fracture, but allow for compatible head across the element 


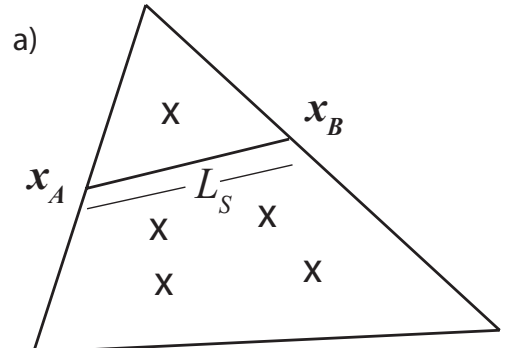

b)

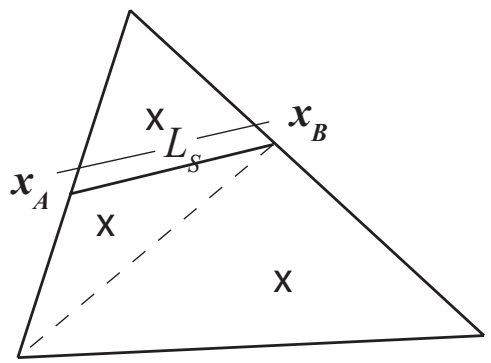

C)

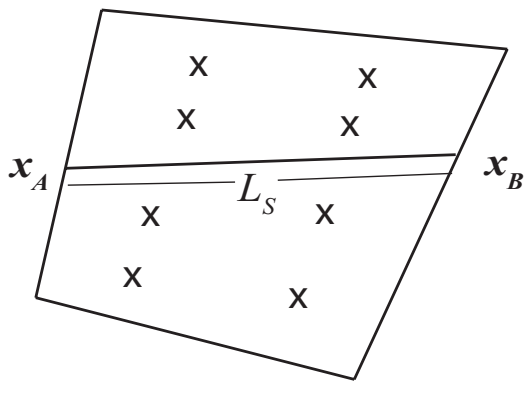

d)

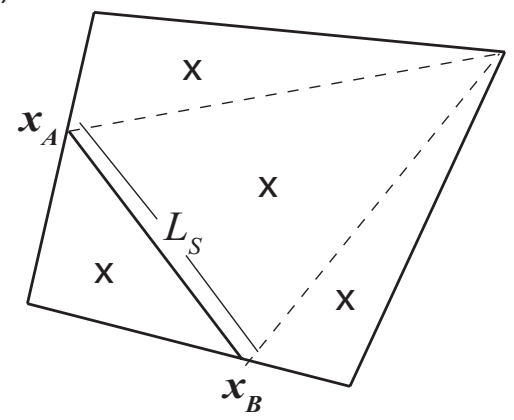

Fig. 1 Examples of elements with linear edges containing enhancements. Nodes are added where the fracture surface intersects the element edges, and the elements are split, with bulk integration being handled separately on each side.

3.1 Element internal flow vector and stiffness matrix

Since we have chosen a piecewise linear interpolation of the head along the fracture, the gradient along the fracture $\hat{\boldsymbol{i}}$ can be written

$$
\hat{\boldsymbol{i}}=\frac{h_{A}-h_{B}}{L_{S}} \frac{\boldsymbol{x}_{A}-\boldsymbol{x}_{B}}{L_{S}}
$$

where the total head at nodes $A$ and $B$ at the ends of the fracture in the element are $h_{A}$ and $h_{B}$, respectively, and $\boldsymbol{x}_{A}$ and $\boldsymbol{x}_{B}$ are the coordinates of those nodes. Hence, the first fraction represents the magnitude of the gradient and the second fraction the direction.

This quantity can be modified as

$$
\hat{\boldsymbol{i}}=\frac{1}{L_{S}^{2}}\left[\left(\boldsymbol{x}_{A}-\boldsymbol{x}_{B}\right) \otimes \boldsymbol{Q}^{e}\right] \boldsymbol{h}^{e}
$$

where 
a)

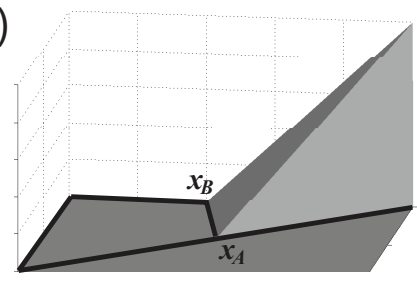

b)

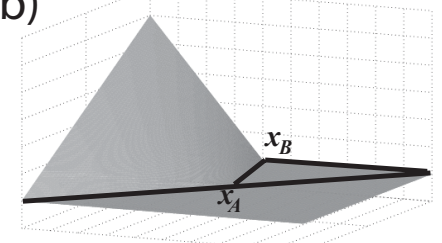

c)

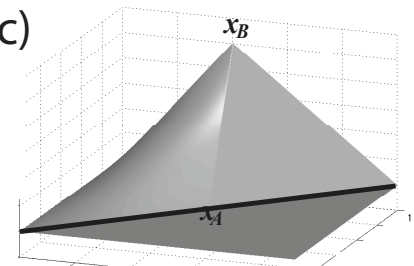

Fig. 2 Three of the shape functions for the chosen element. a) The triangular sub-element uses a linear interpolation on that sub-element, and is zero on the quadratic sub-element for the node that is not adjacent to that sub-element. Similarly, b) the quadratic subelement is bilinear, on that sub-element, and is zero on the triangular sub-element if the node is not adjacent to that sub-element. c) For nodes on both sub-elements, the linear shape function is used on the triangular sub-element, and bilinear of the quadratic sub-element, forming a continuous, though not smooth, shape function

$$
Q^{e}=\left\{\begin{array}{c}
0 \\
\cdot \\
\cdot \\
\cdot \\
0 \\
1 \\
-1
\end{array}\right\} \begin{gathered}
\leftarrow \text { position A } \\
\leftarrow \text { position B }
\end{gathered}
$$

and $\boldsymbol{h}^{e}$ is the element head vector.

The element internal flow vector may then be written as

$$
\begin{aligned}
\boldsymbol{f}_{\text {int }}^{e} & =\int_{\Omega^{e}} \boldsymbol{B}^{e T} \boldsymbol{v} \mathrm{d} \Omega \\
& =\int_{\Omega^{e}} \boldsymbol{B}^{e T}\left\{\boldsymbol{k} \boldsymbol{\nabla} h+\frac{\hat{k}}{L_{S}^{2}}\left[\left(\boldsymbol{x}_{A}-\boldsymbol{x}_{B}\right) \otimes \boldsymbol{Q}^{e}\right] \boldsymbol{h}^{e} \delta_{S}\right\} \mathrm{d} \Omega \\
& =\int_{\Omega^{e}} \boldsymbol{B}^{e T}\left\{\boldsymbol{k} \boldsymbol{B}^{e}+\frac{\hat{k}}{L_{S}^{2}}\left[\left(\boldsymbol{x}_{A}-\boldsymbol{x}_{B}\right) \otimes \boldsymbol{Q}^{e}\right] \delta_{S}\right\} \mathrm{d} \Omega \boldsymbol{h}^{e}
\end{aligned}
$$




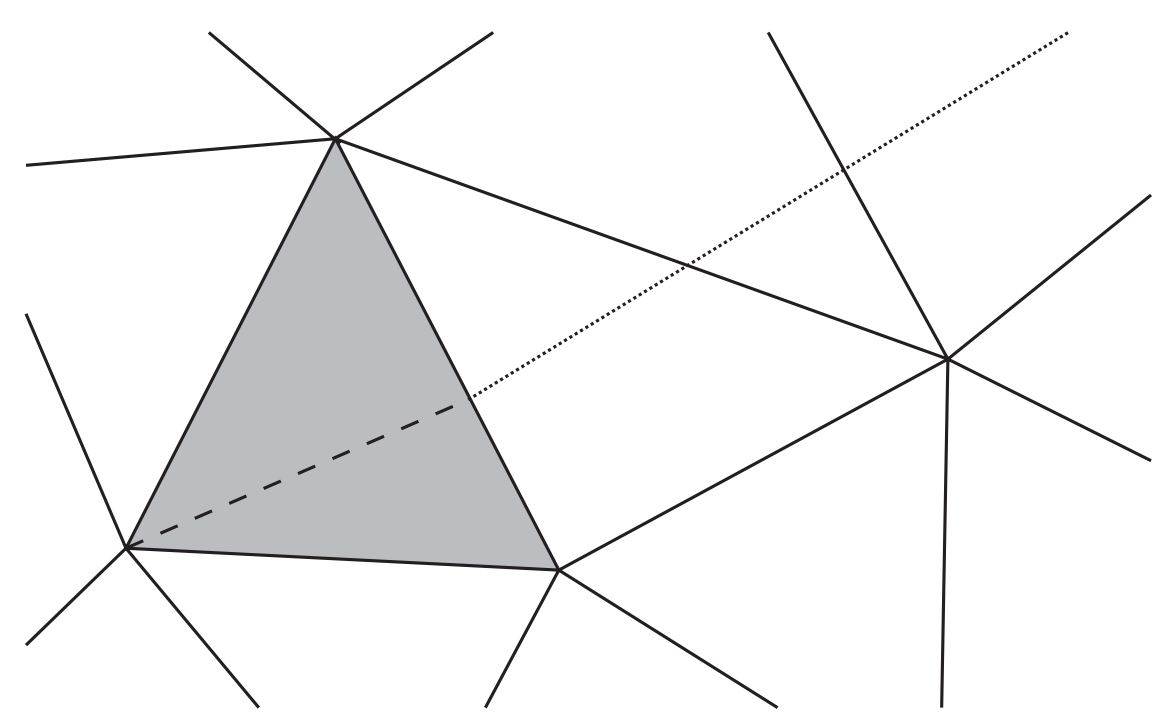

Fig. 3 At the end of a fracture (dotted line), the shaded element must be split in two (dashed line)to maintain continuity of the total head.

$$
=\left\{\int_{\Omega^{e}} \boldsymbol{B}^{e^{T}} \boldsymbol{k} \boldsymbol{B}^{e} \mathrm{~d} \Omega+\int_{S} \boldsymbol{B}^{e T} \frac{\hat{k}}{L_{S}^{2}}\left[\left(\boldsymbol{x}_{A}-\boldsymbol{x}_{B}\right) \otimes \boldsymbol{Q}^{e}\right] \mathrm{d} S\right\} \boldsymbol{h}^{e}
$$

The first term arises from the bulk permeability in the medium, and is equivalent to the internal flow vector for a medium with only bulk permeability obeying Darcy's law. This can be integrated in the standard manner, using Gaussian quadrature on each subelement.

The extra term comes from the flow along the fracture. Since the head is linear on this surface, a single integration point in the center is sufficient to calculate this integral. An issue that arises here is that $\boldsymbol{B}^{e}$ is discontinuous across the surface. However, further analysis of this term reveals that this issue disappears. In two dimensions

$$
\boldsymbol{B}^{e T} \boldsymbol{l}=\left[\begin{array}{cc}
\frac{\partial N_{1}^{e}}{\partial x} & \frac{\partial N_{1}^{e}}{\partial y} \\
\frac{\partial N_{2}^{e}}{\partial x} & \frac{\partial N_{2}^{e}}{\partial y} \\
\ldots & \ldots \\
\frac{\partial N_{n e n-1}^{e}}{\partial x} & \frac{\partial N_{n e n-1}^{e}}{\partial y} \\
\frac{\partial N_{n e n}^{e}}{\partial x} & \frac{\partial N_{n e n}^{e}}{\partial y}
\end{array}\right] \boldsymbol{l}=\left\{\begin{array}{c}
\frac{\partial N_{1}^{e}}{\partial l} \\
\frac{\partial N_{2}^{e}}{\partial l} \\
\ldots \\
\frac{\partial N_{n e n-1}^{e}}{\partial l} \\
\frac{\partial N_{n e n}^{e}}{\partial l}
\end{array}\right\}=\frac{1}{L^{s}}\left\{\begin{array}{c}
0 \\
0 \\
\ldots \\
-1 \\
1
\end{array}\right\}=\frac{1}{L^{s}} \boldsymbol{Q}^{e}
$$

where $\frac{\partial}{\partial l}$ is the derivative in the direction of $\boldsymbol{l}$. For all the nodes not along the discontinuity, the shape function is zero. For the nodes with endpoints on the discontinuity, the shape functions are linear in the direction of the discontinuity path. Therefore the value of the derivative is a constant $\pm 1 / L^{s}$, depending on whether it is increasing or decreasing in the assigned direction of $\boldsymbol{l}$. 
Inserting this into Equation (12), we reach

$$
\boldsymbol{f}_{\text {int }}^{e}=\left\{\int_{\Omega^{e}} \boldsymbol{B}^{e T} \boldsymbol{k} \boldsymbol{B}^{e} \mathrm{~d} \Omega+\int_{S} \frac{\hat{k}}{L_{S}^{3}} \boldsymbol{Q}^{e} \otimes \boldsymbol{Q}^{e} \mathrm{~d} S\right\} \boldsymbol{h}^{e}
$$

Hence, we recover a symmetric formulation for the problem.

As we have already factored out the element head vector, the element stiffness may be easily determined as

$$
\boldsymbol{k}^{e}=\frac{\mathrm{d} \boldsymbol{f}_{i n t}^{e}}{\mathrm{~d} \boldsymbol{h}^{e}}=\int_{\Omega^{e}} \boldsymbol{B}^{e T} \boldsymbol{k} \boldsymbol{B}^{e} \mathrm{~d} \Omega+\int_{S} \frac{\hat{k}}{L_{S}^{3}} \boldsymbol{Q}^{e} \otimes \boldsymbol{Q}^{e} \mathrm{~d} S
$$

The stiffness matrix is symmetric. Again, the first term arises from the bulk permeability, and the second from the enhancement to the permeability along the crack. For the given models, the system of equations is linear, and hence the stiffness matrix is constant. More complex constitutive models will generally result in a nonlinear system of equations.

\subsection{Three-dimensional formulation}

While this article focuses on two-dimensional implementation, the formulation may be extended to three dimensions. In this case, even for the simplest of elements, there is no guarantee that a linear head gradient will exist on the fracture surface, as this would require that the surface is parameterized by only three nodes. In both brick and tetrahedral elements, it is possible that a plane may cut a quadrilateral surface in the element. Still, the hydraulic gradient may be written

$$
\hat{\boldsymbol{i}}=\left(\nabla h \cdot \boldsymbol{l}_{1}\right) \boldsymbol{l}_{1}+\left(\nabla h \cdot \boldsymbol{l}_{2}\right) \boldsymbol{l}_{2}
$$

where $\boldsymbol{l}_{1}$ and $\boldsymbol{l}_{2}$ are mutually orthogonal directions that are also orthogonal to the normal to the fracture surface. In the finite element implementation, this may be written

$$
\begin{aligned}
\hat{\boldsymbol{i}} & =\left[\left(\boldsymbol{B}^{e} \boldsymbol{h}^{e}\right) \cdot \boldsymbol{l}_{1}\right] \boldsymbol{l}_{1}+\left[\left(\boldsymbol{B}^{e} \boldsymbol{h}^{e}\right) \cdot \boldsymbol{l}_{2}\right] \boldsymbol{l}_{2} \\
& =\left(\boldsymbol{l}_{1}^{T} \boldsymbol{B}^{e} \boldsymbol{h}^{e}\right) \boldsymbol{l}_{1}+\left(\boldsymbol{l}_{2}^{T} \boldsymbol{B}^{e} \boldsymbol{h}^{e}\right) \boldsymbol{l}_{2} \\
& =\left(\boldsymbol{l}_{1} \otimes \boldsymbol{l}_{1}\right) \boldsymbol{B}^{e} \boldsymbol{h}^{e}+\left(\boldsymbol{l}_{2} \otimes \boldsymbol{l}_{2}\right) \boldsymbol{B}^{e} \boldsymbol{h}^{e}
\end{aligned}
$$

The internal force vector may then be written 
a)

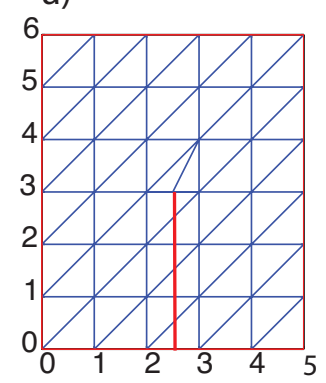

b)

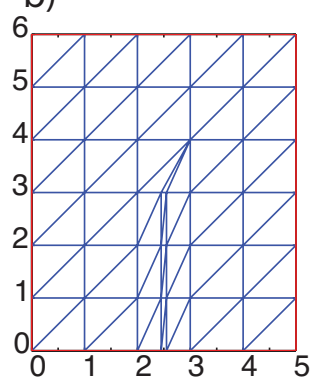

c)

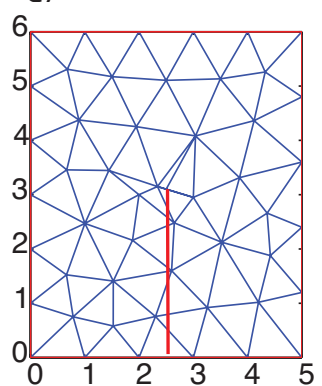

Fig. 4 Rectangular body with a vertical crack. a) with the enhanced elements (fracture in red), b) with a zone of increased permeability as verification, and c) using an unstructured mesh. Refinements of these meshes (not shown) are performed in the standard manner, partitioning each triangle into four new triangles using the midpoint of each edge.

$$
\begin{aligned}
\boldsymbol{f}_{\text {int }}^{e} & \left.=\left\{\int_{\Omega^{e}} \boldsymbol{B}^{e T} \boldsymbol{k} \boldsymbol{B}^{e} \mathrm{~d} \Omega+\int_{S} \hat{k}\left[\boldsymbol{B}^{e T}\left(\boldsymbol{l}_{1} \otimes \boldsymbol{l}_{1}\right) \boldsymbol{B}^{e}+\boldsymbol{B}^{e^{T}}\left(\boldsymbol{l}_{2} \otimes \boldsymbol{l}_{2}\right) \boldsymbol{B}^{e}\right] \mathrm{d} S\right\} 1 \boldsymbol{\otimes} \boldsymbol{\theta}\right) \\
& =\left\{\int_{\Omega^{e}} \boldsymbol{B}^{e T} \boldsymbol{k} \boldsymbol{B}^{e} \mathrm{~d} \Omega+\int_{S} \hat{k}\left[\boldsymbol{Q}_{1} \otimes \boldsymbol{Q}_{1}+\boldsymbol{Q}_{2} \otimes \boldsymbol{Q}_{2}\right] \mathrm{d} S\right\} \boldsymbol{h}^{e}
\end{aligned}
$$

where $\boldsymbol{Q}_{i}=\boldsymbol{l}_{i}^{T} \boldsymbol{B}^{e}$. As in two dimensions, $\boldsymbol{B}^{e}$ may be discontinuous across the surface, but is continuous in the directions along the surface. Hence there is no ambiguity in the definition of $\boldsymbol{Q}_{i}$. The surface integral may be computed with an appropriate number of integration points.

\section{Examples}

\section{1 example 1}

The first example is to verify the model. The problem is a rectangular region, measuring six meters vertically and five horizontally (Figure 4a). The bulk permeability is $5 \times 10^{-5} \mathrm{~m} / \mathrm{s}$. There is a head difference of $21 \mathrm{~m}$ from top to bottom, and no flow is allowed through the sides. There is a fracture that runs vertically from the middle of the bottom of the sample to the centroid of the sample, with $\hat{k}=5 \times 10^{-4} \mathrm{~m}^{2} / \mathrm{s}$. This is verified against the same geometry, but with an area of finite width with increased permeability (Figure $4 \mathrm{~b})$. Initially, this area has a width of $0.1 \mathrm{~m}$ and a permeability of $5 \times 10^{-3}$ $\mathrm{m} / \mathrm{s}$. Multiplied by the cross-sectional area, this will have the same permeability enhancement as the discontinuity, less the small amount of bulk area taken away. The discontinuity is tested with both structured (Figure 4a) and unstructured (Figure 4c) meshes.

The resulting head distributions are shown in Figure 5. The postprocessor does not actually plot the head values on the fracture, reading each element as 
a)

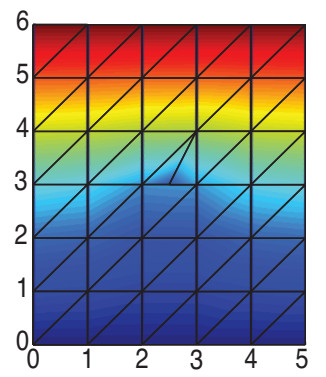

b)

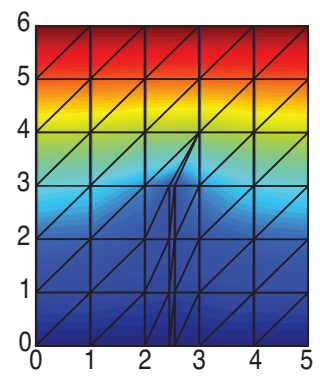

c)

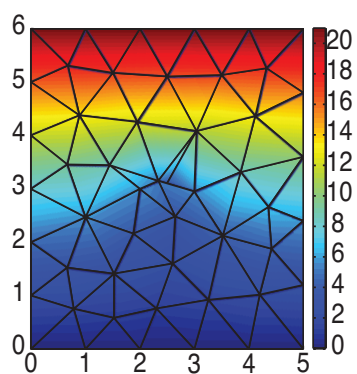

Fig. 5 Total head distributions for the coarsest mesh of each type, showing similar results. The postprocessor does not recognize the added nodes along the element edges.

\begin{tabular}{|c||c|c|c|}
\hline refinement \# & finite area & structured discontinuity & unstructured discontinuity \\
\hline 0 & 5.506 & 5.669 & 5.713 \\
\hline 1 & 5.911 & 6.054 & 5.843 \\
\hline 2 & 6.175 & 6.255 & 6.216 \\
\hline
\end{tabular}

Table 1 Total head one-half meter left of the top of the discontinuity, $m$

\begin{tabular}{|c||c|c|c|}
\hline refinement \# & finite area & structured discontinuity & unstructured discontinuity \\
\hline 0 & 12.04 & 11.96 & 12.09 \\
\hline 1 & 11.76 & 11.68 & 11.89 \\
\hline 2 & 11.62 & 11.57 & 11.62 \\
\hline
\end{tabular}

Table 2 Total flow rate through the sample per unit depth, $\mathrm{cm}^{2} / \mathrm{s}$

a three-node triangle. To verify convergence, we examine two quantities, the head one-half meter to the left of the top of the fracture, and the total flow through the body. These are shown in Tables 1 and 2 respectively. As expected near the discontinuity, some refinement is necessary to get an accurate value of the head. To verify refinement, in the second refinement for the mesh with the finite area of increased permeability, the thickness of that area was halved and the permeability doubled. The three types of meshes converge toward a single result.

The total flow also converges, and more rapidly. As an integrated quantity, it is expected that convergence is more rapid, and coarser meshes give adequate results.

The volumetric flow rate on the crack is shown in Figure 6 for the coarsest mesh and the finest mesh of each type. Since the head is piecewise linear, the flow rate on the crack is constant on each element. Good agreement can be seen comparing the structured, unstructured, and finite width crack approaches, especially on refinement. The fracture picks up more fluid as it moves down, exhibiting square root or logarithmic type increase versus position. 

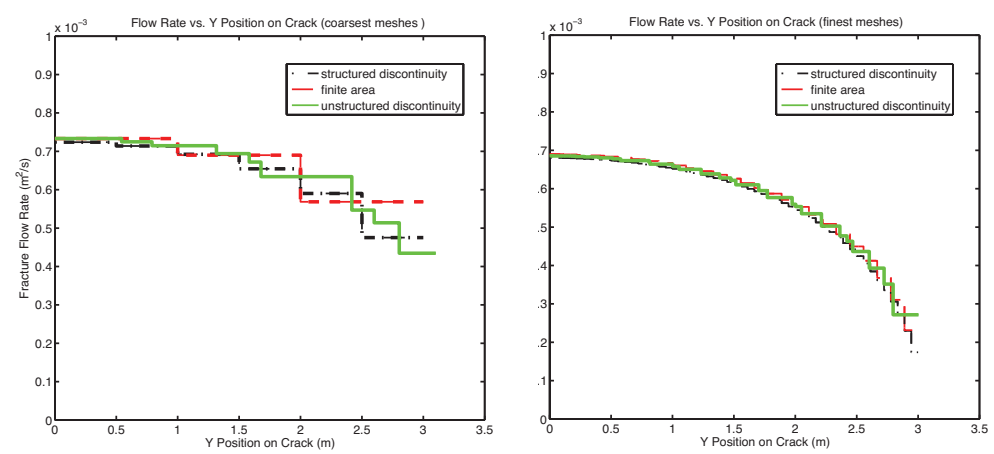

Fig. 6 Enhanced flow on the fracture as a function of position (y-coordinate). The left side shows results for the coarsest mesh of each type, the right for the most refined mesh.

\begin{tabular}{|c||c|c|}
\hline refinement \# & structured discontinuity & unstructured discontinuity \\
\hline 0 & $4.05 \times 10^{-9}$ & $1.49 \times 10^{-9}$ \\
\hline 1 & $1.10 \times 10^{-10}$ & $5.13 \times 10^{-10}$ \\
\hline 2 & $2.27 \times 10^{-12}$ & $1.29 \times 10^{-11}$ \\
\hline
\end{tabular}

Table $3 L^{2}$ norm of volumetric flow rate on crack, $\mathrm{m}^{3} / \mathrm{s}$

\begin{tabular}{|c||c|c|}
\hline refinement \# & structured discontinuity & unstructured discontinuity \\
\hline 0 & 3.47 & 2.93 \\
\hline 1 & 1.31 & 1.68 \\
\hline 2 & 0.668 & 0.901 \\
\hline
\end{tabular}

Table $4 L^{2}$ norm of the error in total head, $\mathrm{m}^{3}$

\begin{tabular}{|c||c|c|}
\hline refinement \# & structured discontinuity & unstructured discontinuity \\
\hline 0 & 2.04 & 2.25 \\
\hline 1 & 1.26 & 1.77 \\
\hline 2 & 0.886 & 1.15 \\
\hline
\end{tabular}

Table $5 L^{2}$ norm of superficial velocity over body, $\times 10^{-4} \mathrm{~m}^{4} / \mathrm{s}$

The error of the flow rate on the crack was studied, compared to a very fine mesh. This is shown in Table 3 .

In addition, the convergence rate for both the structured and unstructured meshes was studied by using a very fine mesh as an "exact" solution. The $L^{2}$ norm of the total head appears to halve as the mesh size is halved, as shown in Table 4.

The overall superficial velocity, being related to gradient of the head, converges somewhat more slowly, but also appears to converge. The error is shown in Table 5 

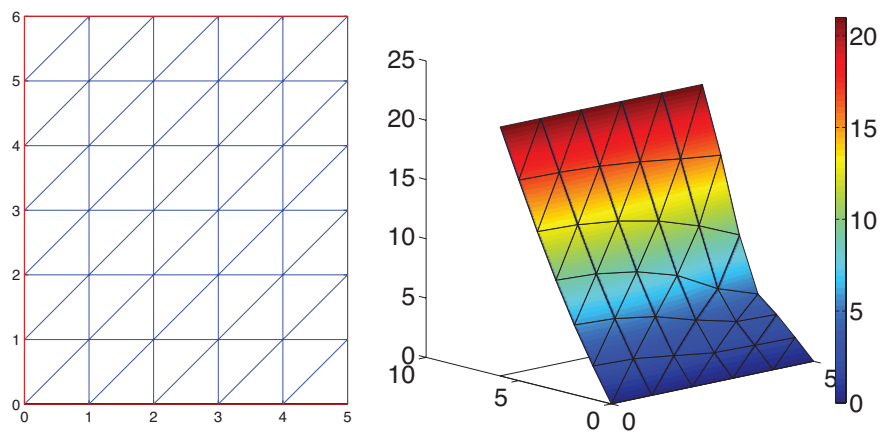

Fig. 7 a) Mesh for body with a diagonal crack. b) Resulting total head in the body

a)

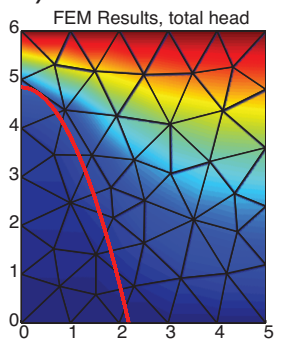

b)

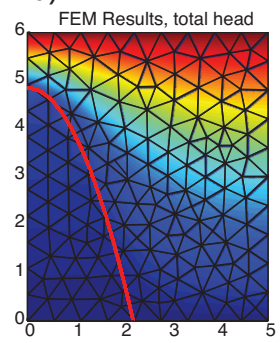

c)

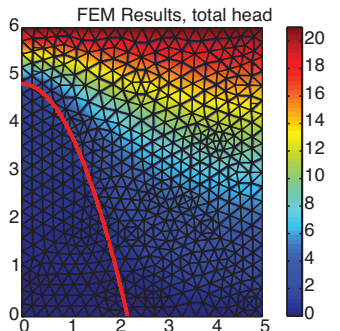

Fig. 8 Example with a curved crack, showing total head in successive refinements of a) 77, b) 287 , and c) 1148 elements

\section{2 example 2}

The second example contains a diagonal crack, as shown in Figure 7a. The crack runs 3.5 meters up the righthand side to 1.5 meters from the bottom. The material properties for both the bulk material and the fracture are the same as in the first example.

The total head (Figure $7 \mathrm{~b}$ ) reveals that much of the potential energy is lost across the fracture, as much of the fluid flows down the fracture. The total flow rate through the sample is $11.62 \mathrm{~cm}^{2} / \mathrm{s}$.

\section{3 example 3}

We extend the formulation to include curved fractures. In the third example the boundaries and boundary conditions are the same, but the crack is a parabolic one extending from the left side to the bottom, as shown in red in Figure 8. Within each element, the crack is considered to have a linear path as developed. This approximation becomes more accurate as the mesh is refined.

The endpoints are found in each element by matching the equation of the curve to the equations of the element edges. In this way, the curve can be 


\begin{tabular}{|c||c|c|}
\hline number of elements & nodal head $(\mathrm{m})$ & total flow rate $\left(\mathrm{m}^{3} / \mathrm{s}\right)$ \\
\hline 77 & 1.4813 & $2.0162 \times 10^{-3}$ \\
\hline 287 & 1.4826 & $2.0236 \times 10^{-3}$ \\
\hline 1148 & 1.475 & $2.0185 \times 10^{-3}$ \\
\hline
\end{tabular}

Table 6 Total head at node at $(0.8926,4.375)$, and total fluid flow through the body

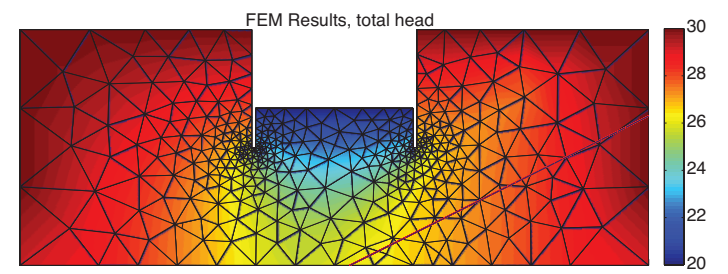

Fig. 9 Geometry and total head calculation for a sheet pile example with a fracture.

tracked as it enters and exits each element, similar to (7). The procedure does not handle the case of a curve exiting and reentering the element, but in practice this case has not been an issue, especially for fine meshes where the curves are close to linear in each element.

The resulting head distribution is shown in Figure 8. The head at a node near the crack, located at $(0.8926,4.375)$, and the total flow for the problem, is shown in Table 6 . Convergence is somewhat slower than for straight cracks, but adequate.

\section{4 example 4}

The final example is an example of fluid flow around a pair of sheet piles, as shown in Figure 9. The sheet piles are 20 meters apart. Inside the piles, the soil has been excavate to 10 meters below the water table, and the resulting water flow is pumped out. The soil has been modeled to 30 meters outside the sheet piles, and it was assumed that the head at that distance was equal to the head at the top of the water table outside of the sheet pile. It was found that increasing the extent of the soil modeled beyond this distance did not significantly affect the solution.

A fracture runs diagonally through the ground as shown. The permeability enhancement is $10^{-3} \mathrm{~m} / \mathrm{s}$, while the bulk permeability is $5 \times 10^{-5} \mathrm{~m} / \mathrm{s}$. The bedrock at the base is considered impermeable.

The resulting head distribution is shown in Figure 9. The total volumetric flow rate in the sheet piles is $4.986 \times 10^{-4} \mathrm{~m}^{3} / \mathrm{s}$. With no fracture, the amount is $4.490 \times 10^{-4} \mathrm{~m}^{3} / \mathrm{s}$. Hence, even without connecting to the exit surface, we can see that localized flow can have a significant influence on overall flow in a system. 


\section{Conclusion}

In summary, we have developed an enhanced finite element for capturing fluid flow through porous media with fracture surfaces. The method allows fractures to be inserted into the model independent of the mesh, making the meshing process simpler. The procedure, by subdividing elements with fractures, is conceptually simpler and easier to implement than an extended or assumed enhanced strain type approach.

The model has been validated by comparing it to a localized zone of finite width. The method compares well to both structured and unstructured meshes. It is worth noting that despite the fact that some sub-elements can have poor aspects ratios, the performance of the method is unaffected.

A mesh modification algorithm has been created which, following the global curve of the mesh, locates the end points of the curve in each element. Within each element, the discontinuity has been considered to be straight. This is an approximation, but as the mesh is refined, the fracture length and path follow more closely the true curve.

This article focuses on Darcy's Law and Darcy-like models for fractured flow. Using this constitutive model results in a linear, symmetric set of equations that are easily solved. The method can, however, be extended to more complex constitutive models, both in the bulk and along the fractured surface. The resulting equations will generally be nonlinear.

Though beyond the scope of this article, the method can be extended to create a jump in the potential field across the discontinuity. This extension would mimic the behavior seen on some fractures, where permeability is inhibited across the crack due to very fine particle size near the localization zone.

The method may also be coupled to mechanical processes, allowing the cross-sectional area of the localized region to change with deformation, bringing about changes in flow rate. Attention will need to be paid to the stability of coupled formulations. Stability in coupled poromechanics has been dealt in several ways, as discussed in $(9 ; 10 ; 18)$, among others. Such extensions are the focus of ongoing research.

Acknowledgements The first author acknowledge the support of the U.S. National Science Foundation, Grant No. CMMI-1030398. Both authors would like to thank two anonymous reviewers for their comments, which improved the manuscript.

\section{References}

1. ANTONELlini, M., AYDIN, A.: EFFECT OF FAULTING ON FLUID-FLOW IN POROUS SANDSTONES - PETROPHYSICAL PROPERTIES. AAPG BULLETIN-AMERICAN ASSOCIATION OF PETROLEUM GEOLOGISTS 78(3), 355-377 (1994) 
2. Barenblatt, G., Zheltov, Y., Kochina, I.: Basic concepts in the theory of seepage of homogeneous liquids in fissured rocks. JOURNAL OF APPLIED MECHANIC AND MATHEMATICS 24(5), 1286-1303 (1960). DOI

3. Belytschko, T., Black, T.: Elastic crack growth in finite elements with minimal remeshing. INTERNATIONAL JOURNAL FOR NUMERICAL METHODS IN ENGINEERING 45(5), 601-620 (1999). DOI 10.1002/(SICI)1097-0207(19990620)45:5;601::AID-NME598¿3.0.CO;2-S

4. Borja, R.I., Koliji, A.: On the effective stress in unsaturated porous continua with double porosity. JOURNAL OF THE MECHANICS AND PHYSICS OF SOLIDS 57(8), 1182-1193 (2009). DOI 10.1016/j.jmps.2009.04.014

5. Callari, C., Armero, F., Abati, A.: Strong discontinuities in partially saturated poroplastic solids. COMPUTER METHODS IN APPLIED MECHANICS AND ENGINEERING 199(23-24), 1513-1535 (2010). DOI 10.1016/j.cma.2010.01.002

6. Foster, C., Mohammad Nejad, T.: Numerical Modeling of Poromechanics in Continuous and Localized Regions. In: Borja, R.I. (ed.) Multiscale and Multiphysics Processes in Geomechanics: Results of the Workshop on Multiscale and Multiphysics Processes in Geomechanics, Stanford, June 2325, 2010, vol. , pp. 141-144. Springer, (2011). DOI

7. Foster, C.D., Borja, R.I., Regueiro, R.A.: Embedded strong discontinuity finite elements for fractured geomaterials with variable friction. International Journal for Numerical Methods in Engineering 72(5), 549-581 (2007). DOI 10.1002/nme.2020

8. J.C. Simo J. Oliver, F.: An analysis of strong discontinuities induced by strain-softening in rate-independent inelastic solids. Computational Mechanics 12, 277 - 296 (1993)

9. Masud, A.: A stabilized mixed finite element method for Darcy-Stokes flow. INTERNATIONAL JOURNAL FOR NUMERICAL METHODS IN FLUIDS 54(6-8), 665-681 (2007). DOI 10.1002/fld.1508. MiniSymposium on Stabilized, Multiscale and Multiphysics Methods, Los Angeles, CA, JUL 16-22, 2006

10. Murad, M., Thomee, V., Loula, A.: Asymptotic behavior of semidiscrete finite-element approximations of Biot's consolidation problem. SIAM JOURNAL ON NUMERICAL ANALYSIS 33(3), 1065-1083 (1996). DOI $10.1137 / 0733052$

11. Noetinger, B., Jarrige, N.: A quasi steady state method for solving transient Darcy flow in complex 3D fractured networks. JOURNAL OF COMPUTATIONAL PHYSICS 231(1), 23-38 (2012). DOI 10.1016/j.jcp.2011.08.015

12. Oliver, J., Huespe, A.E., Samaniego, E., Chaves, E.W.V.: Continuum approach to the numerical simulation of material failure in concrete. International Journal for Numerical and Analytical Methods in Geomechanics $\mathbf{2 8}(7-8), 609-632(2004)$ 
13. Rethore, J., de Borst, R., Abellan, M.A.: A two-scale approach for fluid flow in fractured porous media. INTERNATIONAL JOURNAL FOR NUMERICAL METHODS IN ENGINEERING 71(7), 780-800 (2007). DOI 10.1002/nme.1962

14. Rice, J.R.: Heating and weakening of faults during earthquake slip. JOURNAL OF GEOPHYSICAL RESEARCH-SOLID EARTH 111(B5) (2006). DOI 10.1029/2005JB004006

15. Sun, W., Andrade, J.E., Rudnicki, J.W., Eichhubl, P.: Connecting microstructural attributes and permeability from 3D tomographic images of in situ shear-enhanced compaction bands using multiscale computations. GEOPHYSICAL RESEARCH LETTERS 38 (2011). DOI 10.1029/2011GL047683

16. Tao, Q., Ghassemi, A., Ehlig-Economides, C.A.: A fully coupled method to model fracture permeability change in naturally fractured reservoirs. INTERNATIONAL JOURNAL OF ROCK MECHANICS AND MINING SCIENCES 48(2), 259-268 (2011). DOI 10.1016/j.ijrmms.2010.11.012

17. Therrien, R., Sudicky, E.: Three-dimensional analysis of variably-saturated flow and solute transport in discretely-fractured porous media. JOURNAL OF CONTAMINANT HYDROLOGY 23(1-2), 1-44 (1996). DOI 10.1016/0169-7722(95)00088-7

18. White, J.A., Borja, R.I.: Stabilized low-order finite elements for coupled solid-deformation/fluid-diffusion and their application to fault zone transients. COMPUTER METHODS IN APPLIED MECHANICS AND ENGINEERING 197(49-50), 4353-4366 (2008). DOI 10.1016/j.cma.2008.05.015

19. Yu-jun, Z., Chao-shuai, Y.: Coupled thermo-hydro-mechanical-migratory model for dual-porosity medium and numerical analysis. JOURNAL OF CENTRAL SOUTH UNIVERSITY OF TECHNOLOGY 18(4), 12561262 (2011). DOI 10.1007/s11771-011-0830-3 